\title{
Development of planar Goubau line - microfluidic integrated devices for THz spectroscopy of liquid analytes
}

\author{
M. Swithenbank, ${ }^{a}$ C. Russell, ${ }^{a}$ A. D. Burnett, ${ }^{b}$ L. H. Li, ${ }^{a}$ E. H. Linfield, ${ }^{a}$ A. G. Davies, ${ }^{a}$ \\ J. E. Cunningham ${ }^{\mathrm{a}}$ and C. D. Wood ${ }^{\mathrm{a}}$ \\ ${ }^{a}$ School of Electronic and Electrical Engineering, University of Leeds, Woodhouse Lane, Leeds, LS2 9JT, UK \\ el08m2s@leeds.ac.uk \\ ${ }^{\mathrm{b}}$ The Astbury Centre for Structural and Molecular Biology, University of Leeds, Woodhouse Lane, Leeds, LS2 9JT, UK
}

Keywords: terahertz, PGL, microfluidics, spectroscopy.

\begin{abstract}
We demonstrate the integration of planar Goubau lines with microfluidic systems for the measurement of liquid samples including water and isopropanol. Picosecond pulses are generated and detected by the inclusion of low-temperaturegrown GaAs based photoconductive switches which transmit picosecond pulses along the transmission line. A change in the propagation velocity of pulses is detected and is used to estimate the permittivity of each liquid.
\end{abstract}

\section{Introduction}

Terahertz time-domain spectroscopy (THz-TDS) is of interest in bio-molecular science owing to the femtosecond to nanosecond dynamics that lie at the heart of biological function [1]. Traditionally, free-space THz-TDS has been limited by the strong absorption of water, in which biomolecular materials are often dissolved. On-chip waveguides offer a partial solution to this problem, by supporting travelling-waves on a surface-defined conductor, allowing overlaid liquids to be probed by an evanescent field, with significantly reduced attenuation when compared to freespace systems.

Two types of transmission line have been used for on-chip microfluidic spectroscopy in the frequency range of interest; microstrip lines (MSLs) [2] and planar Goubau lines (PGLs) [3], although coplanar waveguide has also been used at lower (GHz range) frequencies [4]. In previous PGL work [3], microfluidic channels were overlaid perpendicular to a single-wire transmission line, and a vector network analyser (VNA) used to excite the probing electric field. While this geometry allows information about the liquid under test (LUT) to be extracted, here we demonstrate that the reflections resulting from an overlaid microfluidic channel limit the range of solutions that can be analysed in such a system.

In the current work, we integrate microfluidic chips with PGLs for on-chip THz-TDS, taking advantage of their increased extent of evanescent electric field (compared with MSL [5]). Relative to our earlier work [5], we modify the PGL chip design by moving from a transmission to a reflection geometry (see Fig. 1), which permits the same photoconductive switch to record both a reference and sample signal simultaneously, allowing calculation of pulse attenuation and propagation velocity. We use this geometry to measure the time-domain reflections that result from a microfluidic channel bonded perpendicular to the PGL and which interfere with accurate spectroscopic measurements. We next present a novel through-substrate measurement of liquids using devices formed on a thin polyimide (Kapton) film which overcomes this limitation.

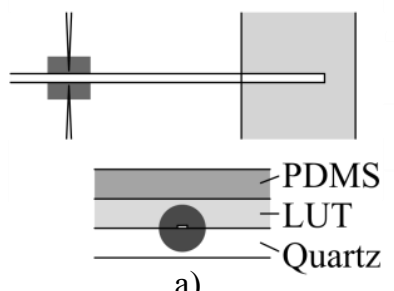

a)

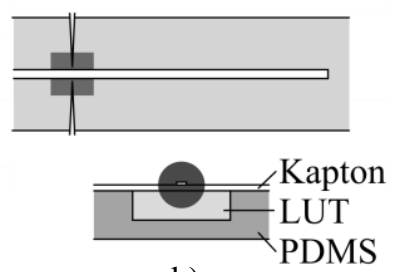

b)
Fig. 1: Schematic (top) and cross-sectional (bottom) diagrams of reflection geometry PGL devices with microfluidic channels incorporated perpendicular to (a), and along the length of (b) the transmission line. Dark-grey squares indicate the position of photoconductive LT-GaAs switches. Dark-grey circles indicate the radial Goubau mode about the centre conductor.

\section{Methodology}

The PGL waveguides were fabricated using a procedure modified from that described in reference [5].

Briefly, a 350-nm-thick layer of low-temperature (LT) GaAs was grown using molecular beam epitaxy on a 100 -nm-thick sacrificial layer of AlAs, itself grown on a $500-\mu \mathrm{m}$-thick semi-insulating (SI) GaAs wafer. Supported by a layer of wax (Wax W, Apiezon), the LT-GaAs was released from the SIGaAs/AlAs structure by selective etching of the AlAs layer in a $\mathrm{HF}: \mathrm{H}_{2} \mathrm{O}$ solution (1:9), and affixed to the target substrate by van Der Waals bonding for five days [6]. Photoconductive switches were defined by slowly etching a single $70 \mu \mathrm{m} \times$ $70 \mu \mathrm{m}$ square into the LT-GaAs for $10 \mathrm{~min}$ using a dilute $\mathrm{H}_{2} \mathrm{SO}_{4}: \mathrm{H}_{2} \mathrm{O}_{2}: \mathrm{H}_{2} \mathrm{O}$ solution (1:8:950). A lithographically patterned layer of Ti:Au (10:150 nm) was deposited to define the PGL and bias arms.

Microfluidic channels were constructed using PDMS (Sylgard 184, Dow Corning) mixed in a 1:10 (curing agent to base) ratio, and poured over a mould defined in $400-\mu \mathrm{m}$-thick SU-8 50 photoresist (selected to be significantly greater than the extent of the evanescent field around the PGL). The cast PDMS was bonded to the substrate using one of two methods depending on the substrate material. 


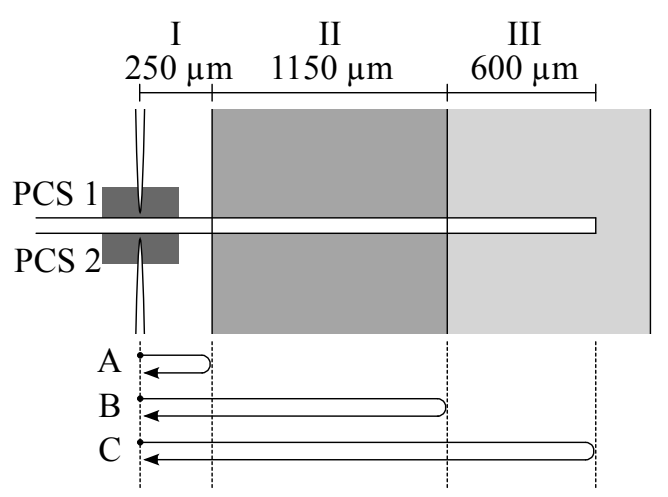

Fig. 2: Schematic of a 2-mm-long reflection PGL geometry on a quartz substrate with an overlaid microfluidic channel. Region I had an air superstrate, region II is the PDMS channel wall, and region III is the channel. Reflections caused by permittivity boundaries are indicated by A, B and C.

Channels were bonded to quartz devices, by a stamp-andstick method [7], (Fig. 1a), in which the PDMS-defined channel was stamped onto a glass slide which had been coated with a layer of wet curing agent, and aligned on the quartz substrate. An irreversible bond was formed between the PDMS and quartz by first degassing in a vacuum oven at $20 \mathrm{mBar}$, and then curing at $80^{\circ} \mathrm{C}$ for one hour.

PDMS channels were bonded to the underside of a Kapton film, as in Fig. 1b, by chemically bonding the two materials [8]. Hydroxyl groups were first formed on the surfaces to be bonded by exposure to an $\mathrm{O}_{2}$-plasma $(50 \mathrm{~W}$, 1 minute). A $2 \%(\mathrm{v} / \mathrm{v})$ aqueous solution of 3-aminopropyltriethoxysilane was then poured over the polyimide film, and a 2\% solution of 3-glycidpropyltriethoxysilane in isopropanol (IPA) was poured over the PDMS channel. The two solutions were left to react for 20 minutes, before being thoroughly washed off in deionised (DI) $\mathrm{H}_{2} \mathrm{O}$, and dried with dry $\mathrm{N}_{2}$. The two surfaces were aligned, brought into contact, and then bonded at room temperature for one hour. A strong amineepoxy bond was formed, which has been shown to withstand flow rates of several hundred channel volumes per hour [8].

Inlet and outlet ports, $2 \mathrm{~mm}$ in diameter, were punched into the PDMS, and silicone pipes were inserted. The elasticity of the PDMS formed a seal around the pipes, which were connected to an automated syringe pump, providing control of the inlet or removal of the LUT.

To generate $\mathrm{THz}$ signals, optical pulses from a Ti:sapphire laser (100 fs duration, $790 \mathrm{~nm}$ centre wavelength, $80 \mathrm{MHz}$ repetition rate and $10 \mathrm{~mW}$ average power) were focused onto PCS 1 , to which a $20 \mathrm{~V}$ bias was applied. The generated pulse then propagated along the PGL, and was reflected from the open circuit stub (see Fig. 2). An optically delayed, mechanically chopped laser beam focused onto PCS 2, allowed the resultant picosecond pulse to be measured using lock-in detection. The TDS response was recorded by measuring the transient photocurrent of this PCS as the arrival time of the optical probe pulses was varied.

\section{Results}

Spectroscopic measurements of IPA and $\mathrm{DI}-\mathrm{H}_{2} \mathrm{O}$ are presented for two reflection-geometry PGL devices, the first with a microfluidic channel overlaid perpendicular to the transmission line, and a second with the channel moved beneath the substrate.

\subsection{Spectroscopy in overlaid channels}

A 2-mm-long reflection geometry PGL was patterned on a $500-\mu \mathrm{m}$-thick quartz substrate. Fig. 3 shows time-domain signals recorded both for the unloaded device, and for the device following bonding of an air-filled PDMS channel, which was arranged perpendicularly to the PGL as illustrated in Fig. 2. Both signals comprised two main features: an initial, 'input' pulse generated by the PCS which has not propagated through the system; and a signal which has propagated along the PGL and reflected back from the opencircuit stub. The effective interaction length between the reflected signal and LUT is $1.2 \mathrm{~mm}$, defined by twice the width of the overlaid fluidic channel. The width of the channel was limited by the need for the PDMS wall to be thick enough to create a quartz-PDMS bond with strength sufficient to prevent leakage, and to leave the LT-GaAs PCSs uncovered to avoid electrical shorts, and unwanted heating effects.

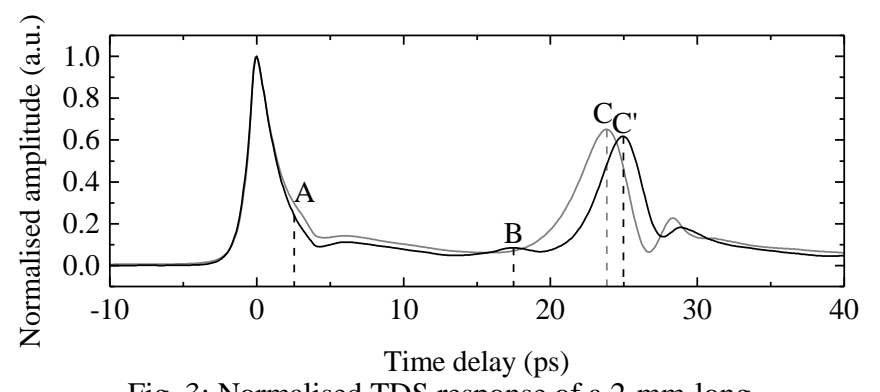

Fig. 3: Normalised TDS response of a 2-mm-long reflection PGL before (grey) and after (black) a PDMS microfluidic channel is bonded to the PGL. Labels represent reflections from the device geometry (Fig. 2).

The PDMS channel introduces a number of interfacial boundaries along the PGL which correspond to changes in the effective impedance of the transmission line (owing to the different permittivities of air and PDMS). The effective permittivity $\left(\varepsilon_{\text {eff }}\right)$ in a given region can be approximated by assuming that the THz-field is radial about the PGL [5], resulting in:

$$
\varepsilon_{\text {eff }}=\frac{\varepsilon_{\text {sub }}+\varepsilon_{\text {sup }}}{2}
$$

where $\varepsilon_{\text {sub }}$ and $\varepsilon_{\text {sup }}$ are the permittivity of the substrate and superstrate, respectively. The quartz permittivity was taken to be 3.8 [9], and so in regions I and III where the superstrate was air, $\varepsilon_{\text {eff }}$ was approximately 2.4 . The permittivity of PDMS was taken to be 2.35 [10], resulting in an $\varepsilon_{\text {eff }}$ for region II of approximately 3.3 .

At a boundary between two regions of differing permittivity, $a$ and $b$, a portion of the energy will be reflected back against the direction of propagation, according to the boundary's reflection coefficient $\Gamma$,

$$
\Gamma=\frac{Z_{\mathrm{eff} b}-Z_{\mathrm{eff} a}}{Z_{\mathrm{eff} b}+Z_{\mathrm{eff} a}} \propto \frac{\varepsilon_{\mathrm{eff} a}-\varepsilon_{\mathrm{eff} b}}{\varepsilon_{\mathrm{eff} a}+\varepsilon_{\mathrm{eff} b}},
$$

where $Z_{\text {eff }}$ and $\varepsilon_{\text {eff }}$ are the effective impedance and permittivity of the respective propagation media. For example, as the permittivity of PDMS is greater than that of air, the increase in permittivity at the boundary between regions I and II 
resulted in the negative reflection A in Fig. 2 and Fig. 3, as confirmed by the proportionality given in equation (2). Conversely, the feature labelled B was a result of the pulse transitioning from the PDMS channel wall into the air-filled cavity.

The positive signals at $\mathrm{C}$ and $\mathrm{C}^{\prime}$ were a result of the pulse reflected from the PGL's open-circuit stub; $\mathrm{C}^{\prime}$ was delayed with respect to $\mathrm{C}$ owing to the increased effective permittivity caused by application of the PDMS channel to the PGL, in accordance with equation (3).

The source of these reflections was further verified by calculating the expected propagation time $t_{\mathrm{g}}$ for the reflected energy to travel a given distance $d$ [11]. The group velocity $v_{\mathrm{g}}$ of a propagating pulse was defined as

$$
v_{\mathrm{g}}=\frac{\mathrm{c}}{\sqrt{\varepsilon_{\mathrm{eff}}}}
$$

from which $t_{\mathrm{g}}$ was calculated as:

$$
t_{\mathrm{g}}=\frac{d}{v_{\mathrm{g}}} .
$$

From equations (3) and (4), $v_{\mathrm{g}}$ in regions I and III was calculated to be $1.94 \times 10^{8} \mathrm{~ms}^{-1}$, and $1.71 \times 10^{8} \mathrm{~ms}^{-1}$ in region II. Therefore, reflections at $\mathrm{A}$ and $\mathrm{B}$ were expected to occur respectively at $2.58 \times 10^{-12} \mathrm{~s}$, and $16.0 \times 10^{-12} \mathrm{~s}$ after the original excitation, which is in reasonable agreement with the results presented in Fig. 3. The expected delay for reflections $\mathrm{C}$ and $\mathrm{C}^{\prime}$ was $20.7 \times 10^{-12} \mathrm{~s}$, and $22.2 \times 10^{-12} \mathrm{~s}$ respectively, approximately $10 \%$ lower than the measured values. This inaccuracy is likely to be caused by the assumption that the Goubau mode is perfectly circular about the centre conductor, which has been shown to be false through simulation [12]. While a more accurate field distribution can be calculated numerically, this problem is yet to be solved analytically.

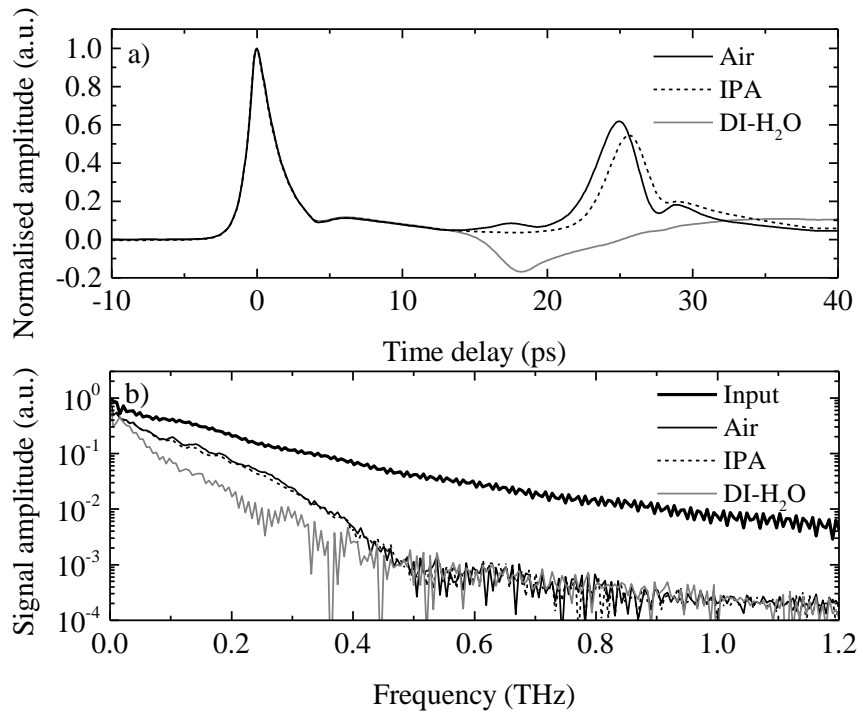

Fig. 4: (a) The time-domain response of a 2-mm-long reflection geometry PGL on a quartz substrate with a microfluidic channel overlaid perpendicular to the transmission line, filled with IPA and DI- $\mathrm{H}_{2} \mathrm{O}$.

(b) The Fourier transform of the time-domain data.

The spectroscopic capabilities of this device were tested by filling the channel with IPA and DI- $\mathrm{H}_{2} \mathrm{O}$. As shown in Fig. 4a, the THz-field was efficiently coupled into the IPA-filled channel, as illustrated by the negligible change in magnitude of the reflection from the PDMS-IPA channel boundary in comparison with the empty channel, suggesting that the two materials have similar permittivities. However, when the channel was filled with $\mathrm{DI}-\mathrm{H}_{2} \mathrm{O}$, the permittivity mismatch resulted in a large negative reflection from the PDMS- $\mathrm{H}_{2} \mathrm{O}$ interface. The strong attenuation of the propagating field by $\mathrm{DI}-\mathrm{H}_{2} \mathrm{O}$ meant that this negative reflection dominated the response, and therefore the majority of the energy measured at PCS 2 had not passed through the LUT.

The frequency responses shown in Fig. $4 \mathrm{~b}$ were calculated by windowing the input and reflected pulses from the data in Fig. 4a. Sample-dependent variation between the time-domain responses in Fig. $4 \mathrm{a}$ began at $13.1 \mathrm{ps,} \mathrm{which} \mathrm{was} \mathrm{therefore}$ chosen as the cut-off for the input window, and the start of the output window. The well-matched IPA sample resulted in a relatively noise-free frequency response. However, while the input pulses remained unchanged, the interference caused by etalons in the output measurement of a mismatched sample such as DI- $\mathrm{H}_{2} \mathrm{O}$ demonstrates a principal limitation of this geometry.

\subsection{Through-substrate spectroscopy}

In order to remove etalons introduced into the time-domain signal by the presence of permittivity boundaries generated by the overlaid fluidic channel, a new arrangement was investigated in which the channel was transferred to the underside of the substrate (see Fig. 1b). In this way, the fluidic channel, and therefore the LUT, could cover the entirety of the PGL without shorting the electrical contacts or interfering with optical excitation of the PCS.

The extent of evanescent field around a PGL of the dimensions used here is approximately $100 \mu \mathrm{m}$ [13]. In order to perform TDS measurements of a sample in a channel located on the underside of the substrate, we therefore require the substrate to be thinner than this value to allow the electric field to interact with an LUT in the channel. Quartz substrates become impractically fragile when thinned to less than $100 \mu \mathrm{m}$, and therefore 2-mm-long reflection-geometry devices were fabricated on a $50-\mu \mathrm{m}$-thick Kapton film.

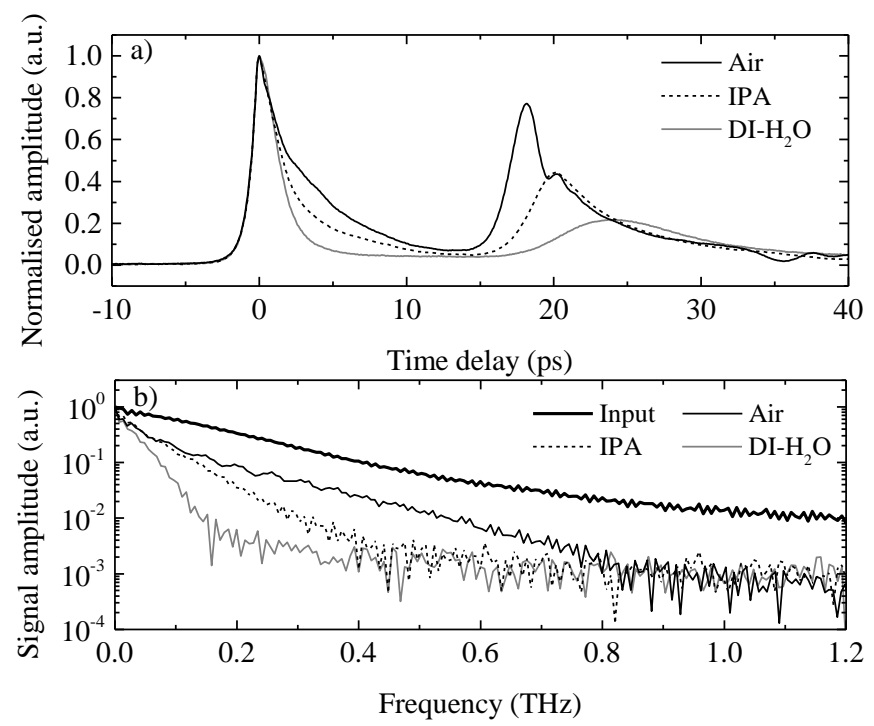

Fig. 5: (a) Normalised TDS of a 2-mm-long reflection PGL with a PDMS microfluidic channel containing air, IPA and DI- $\mathrm{H}_{2} \mathrm{O}$ beneath the substrate. (b) The Fourier transform of the time domain data. 
Repeat measurements of air, IPA and DI- $\mathrm{H}_{2} \mathrm{O}$ were performed using the new geometry and the resulting time-domain traces are shown in Fig. 5. We can see that, by moving the channel beneath the substrate, reflections due to impedance mismatch at material boundaries have been removed. Furthermore, the effective sample interaction length was increased to $4 \mathrm{~mm}$, as the sample now covers the entire length of the $2 \mathrm{~mm}$ PGL.

The increased relaxation time observed in the tail of the input pulse is thought to be a result of heating effects caused by differences in thermal conductivity of the measured liquids $\left(0.025,0.136\right.$, and $0.6 \mathrm{~W} \mathrm{~m}^{-1} \mathrm{k}^{-1}$ for air, IPA and DI- $\mathrm{H}_{2} \mathrm{O}$ respectively), which are now present in the region local to the excitation/detection switches. Despite the broadening in the time-domain, the frequency-domain bandwidth of the generated pulse was not affected.

From the results presented in Fig. 5a, it is possible to approximate the effective permittivity contribution of the LUT across the measured frequency band by consideration of the three-layer system shown in Fig. 1b. Assuming a circular $\mathrm{THz}$ field of radius $r$, with area $A_{\mathrm{THz}}$, the permittivity of the LUT $\varepsilon_{\text {LUT }}$ is [5]

$$
\varepsilon_{\mathrm{LUT}}=\frac{\varepsilon_{\mathrm{eff}} A_{\mathrm{THz}}-\varepsilon_{\mathrm{sup}} A_{\mathrm{sup}}-\varepsilon_{\mathrm{sub}} A_{\mathrm{sub}}}{A_{\mathrm{LUT}}},
$$

where $A_{\text {sup }}, A_{\text {sub }}$, and $A_{\text {LUT }}$ are the respective areas of the superstrate, substrate, and LUT through which the THz field passes. For a substrate of thickness $h$, the areas of intersection can be expressed as

$$
\begin{gathered}
A_{\text {sup }}=\frac{\pi r^{2}}{2}, \\
A_{\text {sub }}=\theta r^{2}+r h \sin \left(\frac{\pi}{2}-\theta\right),
\end{gathered}
$$

and

$$
A_{\mathrm{LUT}}=\frac{\pi-2 \theta}{2} r^{2}-r h \sin \left(\frac{\pi}{2}-\theta\right)
$$

where

$$
\theta=\frac{\pi}{2}-\cos ^{-1}\left(\frac{h}{r}\right)
$$

The time-of-flight of the reflected pulse in the air-filled channel was $18.16 \times 10^{-12} \mathrm{~s}$ which, using equations (3) and (4), result in an $\varepsilon_{\text {eff }}$ of 1.85 . Using equations (5) to (9), the contribution to $\varepsilon_{\text {eff }}$ of the Kapton substrate $\left(\varepsilon_{\text {sub }}\right)$ across the measured frequency band was calculated to be 3.80 . The permittivities of IPA and DI- $\mathrm{H}_{2} \mathrm{O}$ were then calculated to be 3.12 and 8.27 respectively, in agreement with published data for this frequency band ( $\sim 3.0$ and $\sim 8.1$ respectively) [14].

\section{Conclusion}

Two techniques for microfluidic, on-chip time-domain spectroscopy using planar Goubau lines in the terahertz frequency range have been implemented. Problematic reflections resulting from an overlaid microfluidic channel have been removed, by transitioning to a novel throughsubstrate technique, in which the liquid analyte covers the entirety of the PGL, isolated by the substrate.
Expressions for calculating the permittivity of liquid samples in the probed frequency range have been presented, and the results are in good agreement with literature values.

The $4 \mathrm{~mm}$ interaction length achieved with these devices makes them a promising candidate for sensitive measurements of liquids within the microfluidic channel, which would be difficult to measure in free-space THz-TDS transmission systems.

\section{References}

1. Wilmink, G.J. and Grundt, J.E., 'Invited Review Article: Current State of Research on Biological Effects of Terahertz Radiation', J. Infrared. Millim. Te., 2011, 32, (10), pp. 10741122 .

2. Ohkubo, T., Onuma, M., Kitagawa, J., and Kadoya, Y., 'Micro-Strip-Line-Based Sensing Chips for Characterization of Polar Liquids in Terahertz Regime', Appl. Phys. Lett., 2006, 88, (21), p. 212511.

3. Laurette, S., Treizebre, A., Affouard, F., and Bocquet, B., 'Subterahertz Characterization of Ethanol Hydration Layers by Microfluidic System', Appl. Phys. Lett., 2010, 97, (11), p. 111904.

4. Raj, A., Holmes, W.S., and Judah, S.R., 'Wide Bandwidth Measurement of Complex Permittivity of Liquids Using Coplanar Lines', IEEE T. Instrum. Meas., 2001, 50, (4), pp. 905-909.

5. Russell, C., Wood, C.D., Burnett, A.D., Li, L., Linfield, E.H., Davies, A.G., and Cunningham, J.E., 'Spectroscopy of Polycrystalline Materials Using Thinned-Substrate Planar Goubau Line at Cryogenic Temperatures', Lab Chip, 2013, 13, (20), pp. 4065-4070.

6. Cunningham, J., Wood, C., Davies, A., Hunter, I., Linfield, E., and Beere, H., 'Terahertz Frequency Range Band-Stop Filters', Appl. Phys. Lett., 2005, 86, (21), p. 213503.

7. Samel, B., Chowdhury, M.K., and Stemme, G., 'The Fabrication of Microfluidic Structures by Means of FullWafer Adhesive Bonding Using a Poly (Dimethylsiloxane) Catalyst', J. Micromech. Microeng., 2007, 17, (8), pp. 17101714.

8. Tang, L. and Lee, N.Y., 'A Facile Route for Irreversible Bonding of Plastic-Pdms Hybrid Microdevices at Room Temperature', Lab Chip, 2010, 10, (10), pp. 1274-1280.

9. Naftaly, M. and Miles, R.E., 'Terahertz Time-Domain Spectroscopy for Material Characterization', Proc. IEEE, 2007, 95, (8), p. 1658.

10. Khodasevych, I., Shah, C., Sriram, S., Bhaskaran, M., Withayachumnankul, W., Ung, B., Lin, H., Rowe, W., Abbott, D., and Mitchell, A., 'Elastomeric Silicone Substrates for Terahertz Fishnet Metamaterials', Appl. Phys. Lett., 2012, 100, (6), p. 061101.

11. Mittleman, D.M., Hunsche, S., Boivin, L., and Nuss, M.C., 'T-Ray Tomography', Opt. Lett., 1997, 22, (12), pp. 904-906.

12. Treizebré, A., Bocquet, B., Xu, Y., and Bosisio, R.G., 'New Thz Excitation of Planar Goubau Line', Microw. Opt. Techn. Let., 2008, 50, (11), pp. 2998-3001.

13. Russell, C., Wood, C., Dazhang, L., Burnett, A., Li, L., Linfield, E., Davies, A., and Cunningham, J., 'Increasing the Bandwidth of Planar on-Chip Thz Devices for Spectroscopic Applications', in, IRMMW-THz, 2011 36th Int. Conf., (2011) 14. Sato, T. and Buchner, R., 'Dielectric Relaxation Spectroscopy of 2-Propanol-Water Mixtures', J. Chem. Phys., 2003, 118, (10), pp. 4606-4613. 